\title{
Electronic structure of polyhedral carbon cages consisting of hexagons and triangles
}

\author{
A. Ceulemans, * S. Compernolle, A. Delabie, K. Somers, and L. F. Chibotaru \\ Department of Chemistry, University of Leuven, Celestijnenlaan 200F, B-3001 Leuven, Belgium \\ P. W. Fowler \\ School of Chemistry, University of Exeter, Stocker Road, Exeter EX4 4QD, United Kingdom \\ M. J. Margańska and M. Szopa \\ Institute of Physics, Silesian University, ul. Uniwersytecka 4, 40-007 Katowice, Poland \\ (Received 27 December 2000; revised manuscript received 3 April 2001; published 7 March 2002)
}

\begin{abstract}
An infinite series of $(\mathbf{3 , 6})$ cages is defined by trivalent carbon polyhedra composed of hexagonal and four triangular rings. A zone-folding construction is applied to the graphene band structure to yield explicit expressions for the $\pi$-molecular orbitals, energies, and symmetries of the cages that depend only on four indices $m$, $n, p$, and $q$. Leapfrog members of the series $(m-n=0 \bmod 3$ and $p-q=0 \bmod 3)$ have closed shells in a neutral form with two filled nonbonding orbitals; all others have closed shells as dications. Quantum chemical calculations on $\mathrm{C}_{12}, \mathrm{C}_{48}$, and $\mathrm{C}_{52}{ }^{2+}$ confirm this result. Embedding relationships are proved for the spectra of $(3,6)$ cages related by inflation transformations corresponding to stretching and rotation of the polyhedral net.

DOI: 10.1103/PhysRevB.65.115412

PACS number(s): 73.22.-f, 73.20.At, 36.40.-c
\end{abstract}

\section{INTRODUCTION}

The discovery of fullerenes has revived the study of the mathematics of polyhedral cages. Physicists and chemists show a special interest in cages that can be folded from a honeycomb graphitic lattice by introducing pentagonal, triangular, or square defects. In this paper we will be concerned with $(\mathbf{3 , 6})$ cages, i.e., those trivalent polyhedra on $v$ vertices which have four triangular faces, $h=v / 2-2$ hexagonal, and no other faces. The case $h=0$ is a tetrahedron, and otherwise $h \geqslant 4$. All polyhedra in the class have at least $D_{2}$ symmetry, ${ }_{1}^{1}$ but the most symmetrical have tetrahedral ( $T$ or $T_{d}$ ) symmetry, ${ }^{2}$ starting from the tetrahedron itself. In a previous work $^{3}$ it was shown that the Hückel spectrum of a $(\mathbf{3 , 6})$ cage always contains the tetrahedral spectral roots $3,-1,-1$, and -1 , while the remaining eigenvalues are arranged in mirror pairs with respect to the nonbonding level, and various subspectrality relationships hold for cages that are related by inflation transformations. The eigenvalue spectrum of each $(3, \mathbf{6})$ cage was shown to be included in that of the hexagonal covering of the torus. ${ }^{3}$ In addition, González et al. ${ }^{4}$ presented eigenvalue spectra of a special class of highly symmetrical $(\mathbf{3 , 6})$ cages.

In the present paper we will generalize the latter results by providing full expressions for the spectral roots of arbitrary $(3,6)$ cages. Our method is based on an embedding of the spectrum into the highly symmetrical band structure of graphene, following from the embedding of the polyhedra themselves as nets on the graphene sheet.

\section{CONSTRUCTION OF A TETRAHEDRALLY SYMMETRIC $(3,6)$ CAGE}

First we consider a tetrahedrally symmetric $(\mathbf{3 , 6})$ cage. Such a cage can be imagined as a honeycomb lattice inscribed on a master tetrahedron, in such a way that the vertices of this master polyhedron correspond to the centers of the hexagons. Each vertex of the hexagonal lattice is one carbon atom of the cage. The angular defect corresponding to each vertex of the master tetrahedron is $\pi$, and the four vertices accumulate the whole curvature of the surface of the cage and are responsible for four triangular cells in the otherwise hexagonal lattice. The hexagonal lattice can be inscribed in a similar manner on two other Platonic solids: an octahedron (curvature accumulated in six squares of $2 \pi / 3$ angular defect), giving rise to a class of $(\mathbf{4 , 6})$ cages, and an icosahedron (curvature accumulated in 12 pentagons of angular defect $\pi / 3$ ), corresponding to the celebrated fullerene class of $(\mathbf{5 , 6})$ cages. $^{5}$

The primitive cell of the honeycomb lattice of graphite contains two atoms, labeled black and white in Fig. 1(a). Let $\mathbf{T}_{1}$ and $\mathbf{T}_{2}$ be the two generators of the lattice,

$$
\mathbf{T}_{1}=\sqrt{3} a \mathbf{e}_{x}, \quad \mathbf{T}_{2}=\frac{\sqrt{3}}{2} a \mathbf{e}_{x}+\frac{3}{2} a \mathbf{e}_{y},
$$

where $a$ is the nearest-neighbor distance. The triangle $\mathbf{O A B}$ in Fig. 1(a) is the patch of hexagons forming the net of a tetrahedrally symmetric $(\mathbf{3}, \mathbf{6})$ cage unfolded on the plane. Figure 1(b) shows a three-dimensional (3D) plot of the corresponding cage, optimized by a quantum-chemical calculation (vide infra, Sec. VIII).

The patch is an equilateral triangle and therefore, to define the cage, it is sufficient to choose for its side one of the vectors $\mathbf{A}=2 m \mathbf{T}_{1}+2 n \mathbf{T}_{2}$, where $m$ and $n$ are integers, the other at angle of $60^{\circ}$ being $\mathbf{B}=2(m+n) \mathbf{T}_{2}-2 n \mathbf{T}_{1}$. The number of hexagons inside the triangle $\mathbf{O A B}$ is $N / 2$, where $N=4\left(m^{2}+n^{2}+m n\right)$ is the number of its nodes. When folded, four triangles form at the corners of the tetrahedron, leaving $N / 2-2$ full hexagons in the $(\mathbf{3 , 6})$ cage itself. As the cage with $n<m$ is the mirror image of that with $m>n$, it is sufficient to assume that $0 \leqslant n \leqslant m$ with $m>0$. As a result each tetrahedrally symmetric $(\mathbf{3 , 6})$ cage is uniquely determined by the two integers $n$ and $m$. 


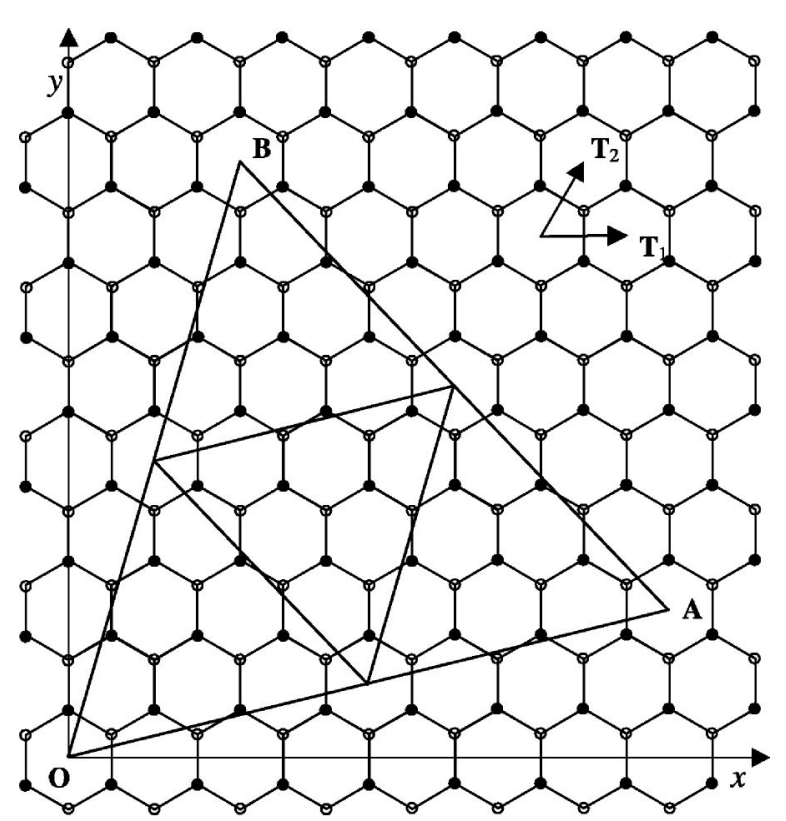

(a)

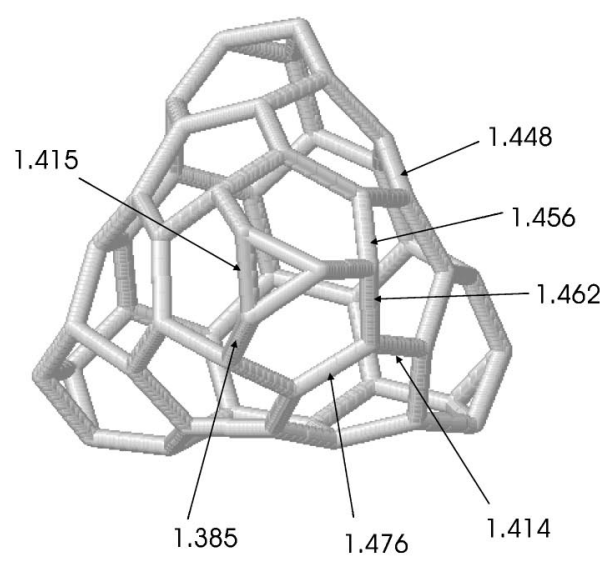

(b)

FIG. 1. Tetrahedral $(\mathbf{3 , 6})$ cage, unfolded on the honeycomb plane (a) and in the folded state (b). Indices correspond to $m=3$ and $n=1$, and the number of carbon atoms is 52 . The folded structure was optimized by a DFT calculation on the dication $\mathrm{C}_{52}{ }^{2+}$.

The Wigner-Seitz primitive cell of the reciprocal space of the honeycomb lattice is the first Brillouin zone of the graphene sheet. It is generated by two vectors $\mathbf{P}_{1}$ $=(2 \pi / \sqrt{3} a) \mathbf{e}_{x}-(2 \pi / 3 a) \mathbf{e}_{y}$, and $\mathbf{P}_{2}=(4 \pi / 3 a) \mathbf{e}_{y}$, as shown in Fig. 2, and it takes the shape of a regular hexagon with vertices

$$
\begin{gathered}
k_{x}= \pm \frac{4 \pi}{3 \sqrt{3} a}, \quad k_{y}=0, \\
k_{x}= \pm \frac{2 \pi}{3 \sqrt{3} a}, \quad k_{y}= \pm \frac{2}{3 a} \pi .
\end{gathered}
$$

Of these six points, only two are linearly independent and belong to the first Brillouin zone (they are $\mathbf{K}_{1}$ and $\mathbf{K}_{2}$ ). Simi-

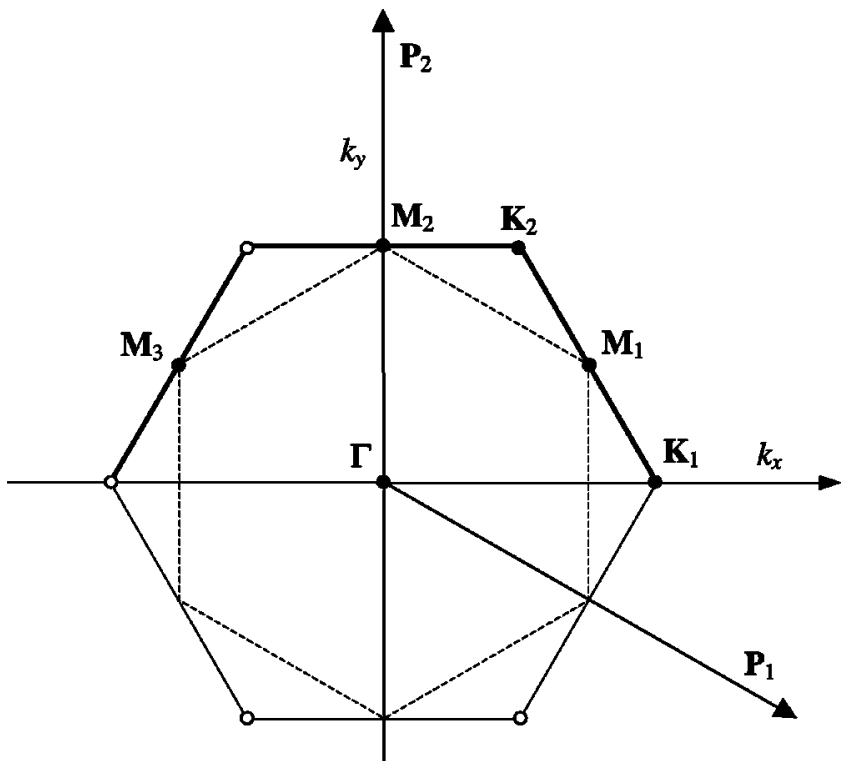

FIG. 2. Wigner-Seitz representation of the first Brillouin zone for the honeycomb lattice. Symmetry points are found at the origin $\boldsymbol{\Gamma}$ (hexagonal), vertices $\mathbf{K}$ (trigonal), and edge centers $\mathbf{M}$ (digonal).

larly, only three edges of the hexagon (we choose those containing $\mathbf{M}_{1}, \mathbf{M}_{2}$, and $\mathbf{M}_{3}$, i.e., the thicker lines in Fig. 2) belong to the zone.

\section{ELECTRONIC STRUCTURE OF GRAPHENE}

In this section we briefly recall the electronic structure of the parent graphene lattice. The wave function of a single electron moving in the potential of the lattice obeys the usual Schrödinger equation

$$
-\frac{\hbar^{2}}{2 m} \nabla^{2} \Psi(\mathbf{r})+V(\mathbf{r}) \Psi(\mathbf{r})=E \Psi(\mathbf{r}),
$$

where $V(\mathbf{r})$ is a periodic potential, i.e., for arbitrary integers $i$ and $j, V\left(\mathbf{r}+i \mathbf{T}_{1}+j \mathbf{T}_{2}\right)=V(\mathbf{r})$. Also note that in our choice of coordinates $V(\mathbf{r})=V(-\mathbf{r})$. The solution of problem (3) in the tight-binding approximation depends on the type of crystallographic lattice, ${ }^{6}$ and is well known. Here we revisit the main points of the derivation for the hexagonal lattice. ${ }^{7}$

The valence structure of graphite is formed by the $\pi$ bands, based on $2 p_{z}$ orbitals which are antisymmetric with respect to reflection through the base plane. In second quantization the $\pi$ electron Hamiltonian of problem (3) is

$$
H=\alpha \sum_{p} a_{p}^{+} a_{p}+\gamma \sum_{\langle p, q\rangle} a_{p}^{+} a_{q},
$$

where $\langle p, q\rangle$ is the summation over nearest neighbors, and $a_{p}^{+}$ and $a_{q}$ are anticommuting fermion operators for $\pi$ orbitals. The constant $\alpha$ is the energy of the electron in a noninteracting $2 p_{z}$ orbital, and $\gamma(\gamma<0)$ is the nearest-neighbor matrix element of the Schrödinger operator with the potential $V$. The solutions of Eq. (4) correspond to irreducible representations $\mathbf{k}$ of the translation group. In order to prepare the subsequent embedding of the tetrahedral lattice, we will dis- 
tinguish the eigenstates further by their parity with respect to the centrosymmetry. This distinction was not made in the standard treatment of Wallace. ${ }^{7}$

The gerade state with even parity is written as

$$
|\mu \mathbf{k}\rangle_{g}=c_{\mu \mathbf{k}}^{(1)}|\mathbf{k}\rangle_{1, g}+c_{\mu \mathbf{k}}^{(2)}|\mathbf{k}\rangle_{2, g}
$$

where

$$
\begin{gathered}
|\mathbf{k}\rangle_{1, g}=\frac{1}{\sqrt{N}} \sum_{p} \exp \left(i s_{p} \mathbf{r}_{p} \cdot \mathbf{k}\right) \mathbf{a}_{p}^{+}|0\rangle, \\
|\mathbf{k}\rangle_{2, g}=\frac{1}{\sqrt{N}} \sum_{p} \exp \left(-i s_{p} \mathbf{r}_{p} \cdot \mathbf{k}\right) \mathbf{a}_{p}^{+}|0\rangle .
\end{gathered}
$$

In Eq. (5), $\mu=1$ and 2 is the band index, the summation over $p$ in Eq. (6) is over all the $N$ atoms at positions $\mathbf{r}_{p}, s_{p}=1$ for black atoms and $s_{p}=-1$ for white atoms, and $\mathbf{a}_{p}^{+}|0\rangle$ is the atomic orbital centered at atom $p$.

The coefficients of the even wave functions [Eq. (5)] are found to be

$$
c_{\mu \mathbf{k}}^{(1)}=\frac{1}{\sqrt{2}}\left(\frac{\overline{\lambda_{\mathbf{k}}}}{\left|\lambda_{\mathbf{k}}\right|}\right)^{1 / 2}, \quad c_{\mu \mathbf{k}}^{(2)}=-\frac{(-1)^{\mu}}{\sqrt{2}}\left(\frac{\lambda_{\mathbf{k}}}{\left|\lambda_{\mathbf{k}}\right|}\right)^{1 / 2},
$$

where

$$
\lambda_{\mathbf{k}}=\exp \left(-i k_{y} a\right)+2 \cos \left(\frac{\sqrt{3}}{2} k_{x} a\right) \exp \left(i \frac{k_{y} a}{2}\right) .
$$

On the other hand, the ungerade state of odd parity is

$$
|\mu \mathbf{k}\rangle_{u}=d_{\mu \mathbf{k}}^{(1)}|\mathbf{k}\rangle_{1, u}+d_{\mu \mathbf{k}}^{(2)}|\mathbf{k}\rangle_{2, u}
$$

where

$$
\begin{gathered}
|\mathbf{k}\rangle_{1, u}=\frac{1}{\sqrt{N}} \sum_{p} s_{p} \exp \left(i s_{p} \mathbf{r}_{p} \cdot \mathbf{k}\right) \mathbf{a}_{p}^{+}|0\rangle, \\
|\mathbf{k}\rangle_{2, u}=\frac{1}{\sqrt{N}} \sum_{p} s_{p} \exp \left(-i s_{p} \mathbf{r}_{p} \cdot \mathbf{k}\right) \mathbf{a}_{p}^{+}|0\rangle .
\end{gathered}
$$

For this odd wave function [Eq. (9)], one has

$$
d_{\mu \mathbf{k}}^{(1)}=c_{\mu \mathbf{k}}^{(1)}, \quad d_{\mu \mathbf{k}}^{(2)}=-c_{\mu \mathbf{k}}^{(2)} .
$$

For every pair of antipodal points $\mathbf{k}=\left(k_{x}, k_{y}\right)$ and $-\mathbf{k}=\left(-k_{x},-k_{y}\right)$ in the Brillouin zone, one may construct in each band one even eigenstate and one odd eigenstate, which each have the same eigenenergy $E_{\mu \mathbf{k}}$;

$$
\begin{aligned}
E_{\mu \mathbf{k}}= & \alpha-(-1)^{\mu} \\
& \times \gamma\left(1+4 \cos ^{2} \frac{\sqrt{3}}{2} k_{x} a+4 \cos \frac{\sqrt{3}}{2} k_{x} a \cos \frac{3}{2} k_{y} a\right)^{1 / 2} .
\end{aligned}
$$

From Eq. (12) it is clear that the two bands corresponding to $\mu=1$ and 2 are symmetric with respect to the energy $E$ $=\alpha$. The splitting of the two bands is $6 \gamma$ at the center $\boldsymbol{\Gamma}$ of the zone, and then decreases toward the edges. At the points
$\mathbf{M}_{1}, \mathbf{M}_{2}$, and $\mathbf{M}_{3}$ it is $2 \gamma$, and at $\mathbf{K}_{1}$ and $\mathbf{K}_{2}$ (and only there) the splitting vanishes. At half-filling of the band the number of electrons is equal to the number of atoms in the cage. The Fermi energy is $E=\alpha$, which means that in the first Brillouin zone the Fermi surface consists of just two isolated Fermi points $\mathbf{K}_{1}$ and $\mathbf{K}_{2}$. To simplify further discussion, we will express all energies with respect to the Fermi level, by putting $\alpha=0$.

Exceptional points with respect to the centrosymmetry of the Brillouin zone are the center of the zone $\boldsymbol{\Gamma}$ and the centers of the edges, $\mathbf{M}_{1}, \mathbf{M}_{2}$, and $\mathbf{M}_{3}$, all of which coincide with centers of symmetry. In the $\mu=1$ band the $\boldsymbol{\Gamma}$ point is an even eigenstate, corresponding to the totally bonding combination, while the eigenstates in the three $\mathbf{M}$ points are all odd. The opposite holds for the $\mu=2$ band: here the $\Gamma$ point is a single odd, totally antibonding eigenstate, and the three $\mathbf{M}$ points are even combinations. The $\mathbf{K}_{1}$ and $\mathbf{K}_{2}$ points are also special, since these are the only points for which $\lambda_{\mathbf{k}}$ $=0$, and as a result the interaction element between $|\mathbf{k}\rangle_{1, g}$ and $|\mathbf{k}\rangle_{2, g}$ vanishes, and similarly for $|\mathbf{k}\rangle_{1, u}$ and $|\mathbf{k}\rangle_{2, u}$. At $E=0$ there are thus four solutions, two of even parity with eigenvectors $\left|\mathbf{K}_{1}\right\rangle_{1, g}$ and $\left|\mathbf{K}_{1}\right\rangle_{2, g}$, and two of odd parity with eigenvectors $\left|\mathbf{K}_{1}\right\rangle_{1, u}$ and $\left|\mathbf{K}_{1}\right\rangle_{2, u}$. Substituting $\mathbf{K}_{1}$ with $\mathbf{K}_{2}$ does not yield further solutions, since $\mathbf{K}_{1}$ and $\mathbf{K}_{2}$ are related to each other by centrosymmetry: $\mathbf{K}_{2}=-\mathbf{K}_{1}+\mathbf{P}_{1}+\mathbf{P}_{2}$. $\left|\mathbf{K}_{1}\right\rangle_{2, g}$ and $\left|\mathbf{K}_{2}\right\rangle_{1, g}$ are thus identical within a constant phase factor.

\section{BOUNDARY CONDITIONS FOR THE COVERING FUNCTION}

In this section we will show that the energy spectrum of any tetrahedrally symmetric $(\mathbf{3 , 6})$ cage can be derived from the spectrum of the graphene lattice by an appropriate choice of the boundary conditions.

In case of a tetrahedrally symmetric $(\mathbf{3 , 6})$ cage, the wave function, solving Eq. (3), should obey the boundary conditions, required by matching of the function in value and gradient at edge points brought together in the gluing of the net,

$$
\begin{gathered}
\psi(t \mathbf{A})=\psi(\mathbf{A}-t \mathbf{A}), \\
\psi(t \mathbf{B})=\psi(\mathbf{B}-t \mathbf{B}), \\
\psi[\mathbf{A}+t(\mathbf{B}-\mathbf{A})]=\psi[\mathbf{B}+t(\mathbf{A}-\mathbf{B})], \\
\mathbf{n} \cdot \boldsymbol{\nabla} \psi(t \mathbf{A})=-\mathbf{n} \cdot \boldsymbol{\nabla} \psi(\mathbf{A}-t \mathbf{A}), \\
\mathbf{n} \cdot \boldsymbol{\nabla} \psi(t \mathbf{B})=-\mathbf{n} \cdot \boldsymbol{\nabla} \psi(\mathbf{B}-t \mathbf{B}), \\
\mathbf{n} \cdot \boldsymbol{\nabla} \psi[A+t(\mathbf{B}-\mathbf{A})]=-\mathbf{n} \cdot \boldsymbol{\nabla} \psi[\mathbf{B}+t(\mathbf{A}-\mathbf{B})],
\end{gathered}
$$

where $t$ is an arbitrary parameter, $0 \leqslant t<\frac{1}{2}$ and $\mathbf{n}$ denotes the outward normal unit vector on the patch boundary. The wave function $\psi$ of the cage is restricted to the area of the patch $\mathbf{O A B}$. Now we can construct the covering function $\Psi$, which is also a solution of Eq. (3), but defined on the whole graphene lattice in such a way that, when restricted to the patch, it coincides with the wave function of the unfolded tetrahedron 


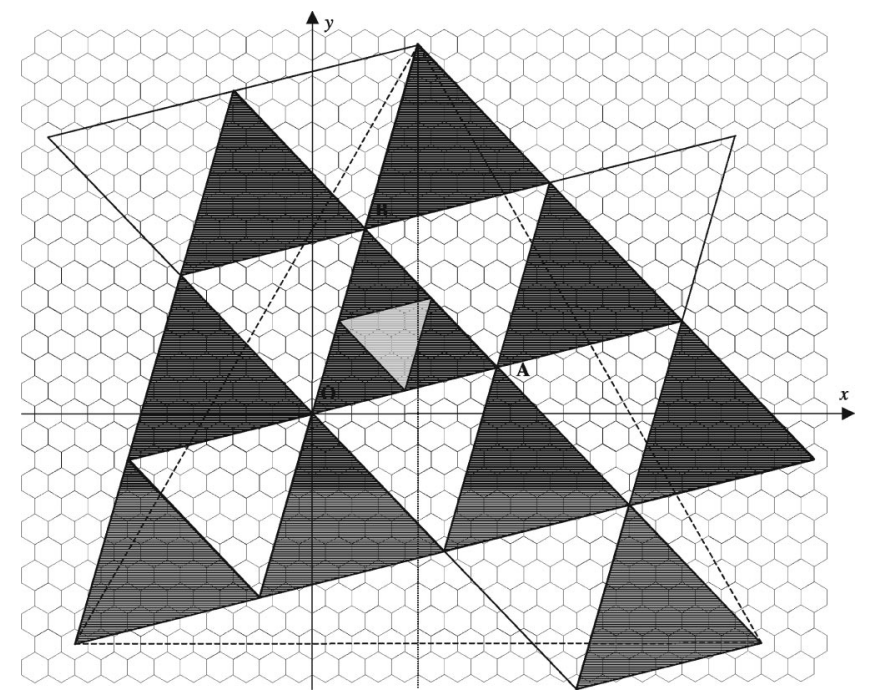

FIG. 3. Triangular tiling of the honeycomb plane by the patch $\mathbf{O A B}$. Black tiles are generated by translations along $\mathbf{O A}$ and $\mathbf{O B}$. Centrosymmetry in $\mathbf{O}$ maps black tiles onto white ones. The dashed lines represent waves propagating in a direction perpendicular to the bonds, with wavelength $d_{\perp}$. The vertical dotted line is in a direction parallel to the bonds, with wavelength $d_{\|}$.

$$
\left.\Psi\right|_{\text {OAB }}(\mathbf{r})=\psi(\mathbf{r}) .
$$

Note that, because of the identification of points $\mathbf{O}, \mathbf{A}$, and $\mathbf{B}$ on the tetrahedron, $\Psi(\mathbf{O})=\Psi(\mathbf{A})=\Psi(\mathbf{B})$. Moreover, it is no restriction on $\psi$ to assume that for an arbitrary vector $\mathbf{r}$,

$$
\Psi(\mathbf{r})=\Psi(\mathbf{r}+\mathbf{A}) \quad \text { and } \quad \Psi(\mathbf{r})=\Psi(\mathbf{r}+\mathbf{B}),
$$

i.e., that $\Psi$ is periodic with vector periods $\mathbf{A}$ and $\mathbf{B}$, and hence

$$
\Psi(\mathbf{r})=\Psi(\mathbf{r}+i \mathbf{A}+j \mathbf{B})
$$

for arbitrary integers $i$ and $j$. Equation (16) extends the solution $\psi$ from the unfolded tetrahedron to a half-tiling of the plane, represented by shaded triangles in Fig. 3. The full covering function of $\psi$ is given by assuming that, in addition to Eqs. (15), $\Psi$ is symmetric with respect to inversion, i.e.,

$$
\Psi(\mathbf{r})=\Psi(-\mathbf{r}) .
$$

Condition (17) extends the definition of $\Psi$ to those tiles not accessible by translation (white triangles in Fig. 3). Moreover, conditions (15) and (17) imply that the function $\psi$ obeys boundary conditions (13). Indeed, by the consecutive use of Eqs. (17) and (15) we have

$$
\Psi(t \mathbf{A})=\Psi(-t \mathbf{A})=\Psi(\mathbf{A}-t \mathbf{A}),
$$

i.e., the first equation of Eqs. (13). Similarly one can prove the fourth equation of Eq. (13)

$$
\mathbf{n} \cdot \boldsymbol{\nabla} \Psi(t \mathbf{A})=n \cdot(-\boldsymbol{\nabla}) \Psi(-t \mathbf{A})=-n \cdot \boldsymbol{\nabla} \Psi(\mathbf{A}-t \mathbf{A}) .
$$

The remaining boundary conditions of Eq. (13) can be obtained in the same way. On the other hand, having a solution $\Psi$ of Eq. (3), one can always construct from it a form
$(1 / \sqrt{2})[\Psi(\mathbf{r})+\Psi(-\mathbf{r})]$, which is symmetric with respect to inversion (17). This is a direct consequence of the centrosymmetry of $V(r)$ in our choice of coordinates. Summarizing, we have shown that the existence of a covering function $\Psi$, obeying the Schrödinger equation (3) with boundary conditions (15) and (17) on the whole plane, is a necessary and sufficient condition for the existence of the wave function $\psi$ that obeys the Schrödinger equation (3) with boundary conditions (13) on the unfolded tetrahedron. One can conclude that those orbitals of graphene which obey conditions (15) and (17) will be eigenstates of the tetrahedrally symmetric $(\mathbf{3 , 6})$ cage.

\section{ELECTRONIC EIGENFUNCTIONS OF A $(3,6)$ CAGE}

It is not difficult to find eigenfunctions for the graphene lattice which are simultaneously eigenfunctions of the tetrahedrally symmetric $(\mathbf{3 , 6})$ cage. Periodic boundary conditions in two independent directions $\mathbf{A}$ and $\mathbf{B}$ give rise to a twodimensional grid of allowed $\mathbf{k}$ vectors in the Brillouin zone. As shown by Ceulemans et al. ${ }^{8}$ this zone-folding procedure yields precisely the spectrum of a toroidal polyhex structure, based on the parallellogram patch with sides $\mathbf{O A}$ and $\mathbf{O B}$. The allowed $\mathbf{k}$ vectors, which guarantee the periodicity, obey

$$
\begin{aligned}
& \mathbf{k} \cdot \mathbf{A}=2 \pi l_{1}, \\
& \mathbf{k} \cdot \mathbf{B}=2 \pi l_{2},
\end{aligned}
$$

where $l_{1}$ and $l_{2}$ are integers counting allowed eigenstates of the cage and defining allowed momentum vectors

$$
\begin{gathered}
k_{x}=\frac{4 \pi}{\sqrt{3} a N}\left[(m+n) l_{1}-n l_{2}\right], \\
k_{y}=\frac{4 \pi}{3 a N}\left[(n-m) l_{1}+(2 m+n) l_{2}\right] .
\end{gathered}
$$

This result, in the particular case $m=n$, was obtained by Gonzalez et al. ${ }^{4}$ The number of momenta in the first Brillouin zone is $N$, i.e., the number of atoms of the original lattice. The first Brillouin zone for the cage shown in Fig. 1, i.e., corresponding to $m=3$ and $n=1$, is shown in Fig. 4(a). The additional condition [Eq. (17)] now requires that only even eigenstates are selected. This effectively halves the number of states in the torus by selecting only those which are symmetric with respect to inversion through the midpoint of the line AB. Because of periodicity conditions (13), such solutions will also be symmetric with respect to the midpoints of the lines $\mathbf{O B}$ and $\mathbf{O A}$.

In this way the gluing conditions are satisfied along all the open edges of the unfolded tetrahedral patch, precisely as required for tetrahedral eigenstates. The selection of even states from the grid points operates in the same way as for the full Brillouin zone. Every antipodal pair of grid points yields an even eigenstate in each band, while the $\boldsymbol{\Gamma}$ and $\mathbf{M}$ points yield even states in the $\mu=1$ and 2 bands, respectively. For the $\mathbf{K}$ points there are two even states, owing to the degeneracy of the two bands. The special states of the first Brillouin zone, $\boldsymbol{\Gamma}, \mathbf{M}_{1}, \mathbf{M}_{2}$, and $\mathbf{M}_{3}$, are always al- 

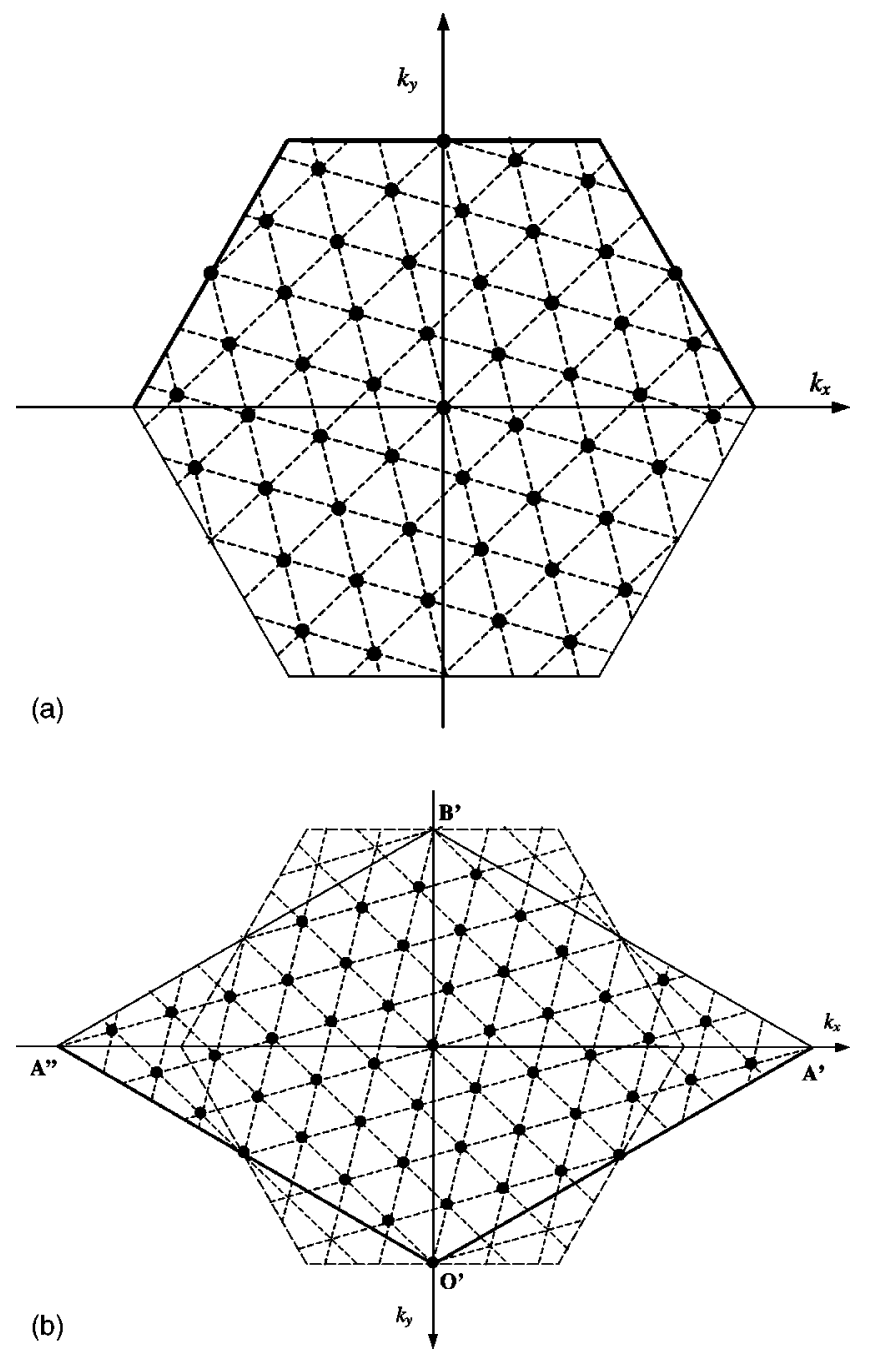

FIG. 4. Allowed $\mathbf{k}$ states for a $(3,1)$ cage in the first Brillouin zone. The $\mathbf{k}$ states form a centrosymmetric triangular grid (a) which includes $\boldsymbol{\Gamma}$ and $\mathbf{M}$ points. For all other points each pair of antipodal points yields one allowed even eigenstate. A direct graphical construction of this grid is shown in (b). Starting from the original patch $\mathbf{O A B}$ in direct space, we take its dual. This is the triangle $\mathbf{O}^{\prime} \mathbf{A}^{\prime} \mathbf{B}^{\prime}$, and we then apply central symmetry to form the rhombus $\mathbf{O}^{\prime} \mathbf{A}^{\prime} \mathbf{B}^{\prime} \mathbf{A}^{\prime \prime}$. The mirror image of this rhombus forms an elementary cell of reciprocal space, equivalent to the first Brillouin zone in (a), where its $k_{x}$ axis is taken along the longer diagonal and its $k_{y}$ axis along the shorter one. (b) shows the reflected elementary cell, as is clear from the opposite directions of the $k_{y}$ axis in (a) and (b).

lowed momenta of the tetrahedral cage, irrespective of $m$ and $n$. The point $\boldsymbol{\Gamma}$ corresponds to the lowest energy state $E$ $=3 \gamma$, and its wave function is homogeneous over the cage. The momenta $\mathbf{M}_{1}, \mathbf{M}_{2}$ and $\mathbf{M}_{3}$ belong to the triply degenerate level $-\gamma$, and represent a wavelength $3 a$.

The periodicity of the wave function in two vectors $\mathbf{A}$ and $\mathbf{B}$ is a consequence of the construction of the covering function $\Psi$. One can, however, ask a natural question: is the wave function $\Psi$ also periodic in some vectors that are parallel or perpendicular to bond directions of the hexagonal lattice? The answer is affirmative. Indeed, the three dashed lines in Fig. 3 represent the periods of the triangular tiling in the directions perpendicular to the bonds in case of the $m=3$, $n=1$ lattice. More generally one can show, that the period of the tiling of the $m, n$ cage in directions $\mathbf{e}_{\perp}^{(1)}=\mathbf{e}_{x}, \mathbf{e}_{\perp}^{(2)}=\frac{1}{2} \mathbf{e}_{x}$ $+(\sqrt{3} / 2) \mathbf{e}_{y}$ and $\mathbf{e}_{\perp}^{(3)}=-\frac{1}{2} \mathbf{e}_{x}+\sqrt{3} / 2 \mathbf{e}_{y}$, i.e., perpendicular to the bonds, is

$$
d_{\perp}=\frac{2 \sqrt{3} a\left(m^{2}+n^{2}+m n\right)}{\mathcal{G}(m, n)},
$$

where $\mathcal{G}(m, n)$ is the greatest common divisor of $m$ and $n$. In the case of the $m=3, n=1$ lattice in Fig. $3, d_{\perp}=26 \sqrt{3} a$. Note that a path which starts in an arbitrary point of the tetrahedron and leads in one of the directing $\mathbf{e}_{\perp}^{(i)}$ may run several times around the tetrahedron before it returns to the starting point (dashed line in Fig. 3). The wavelength function propagating in this direction must be commensurate with the total length of this path. More precisely, a condition analogous to Eq. (20) must hold

$$
\mathbf{k} \cdot d_{\perp} \mathbf{e}_{\perp}^{(i)}=2 \pi l,
$$

where $i=1,2$, and 3 , and $l$ is an arbitrary integer. In the case of $\mathbf{K}_{1}$ and $\mathbf{K}_{2}$ vectors (where the wave functions must propagate in directions perpendicular to the bonds) Eq. (23) yields either

$$
l=\frac{2}{3} \frac{m^{2}+n^{2}+m n}{\mathcal{G}(m, n)}
$$

or twice this value depending on whether the directions of $\mathbf{K}_{1}$ or $\mathbf{K}_{2}$ and $\mathbf{e}_{\perp}^{(i)}$ are respectively at an angle of $60^{\circ}$ or $0^{\circ}$. It is easy to show, that $l$ is an integer provided that 3 is a divisor of $m-n$ and so the necessary condition for the $\mathbf{K}_{1}$ and $\mathbf{K}_{2}$ vectors to belong to the first Brillouin zone is that the parameters of the lattice $m, n$ obey this divisibility condition.

Similar considerations show that $\Psi$ must be periodic in the directions parallel to the bonds (i.e., directions along which the $\mathbf{M}$ waves propagate). The period for an arbitrary cage is $d_{\|}=\sqrt{3} d_{\perp}$, but $d_{\|}=d_{\perp} / \sqrt{3}$ for a cage where 3 is a divisor of $m-n$. In case of a cage with $m=3, n=1$, and $d_{\|}=78 a$, half of the period is drawn as a vertical, dotted line in Fig. 3. The period $d_{\|}$is always a multiple of $3 a$ (the wavelength of the $\mathbf{M}$ states). This is consistent with our earlier observation from Eq. (21), that $\mathbf{M}$ points are allowed momentum vectors for all $(n, m)$.

The allowed $\mathbf{k}$ states form a triangular lattice like the dual of the hexagonal lattice of the tetrahedron. A graphical construction of the reciprocal lattice is shown in Fig. 4(b). The rhombus $\mathbf{O}^{\prime} \mathbf{A}^{\prime} \mathbf{B}^{\prime} \mathbf{A}^{\prime \prime}$ in this figure is an elementary cell in the reciprocal lattice equivalent to the first Brillouin zone from Fig. 4(a). It can be seen that both equilateral triangles $\mathbf{O}^{\prime} \mathbf{A}^{\prime} \mathbf{B}^{\prime}$ and $\mathbf{O}^{\prime} \mathbf{A}^{\prime \prime} \mathbf{B}^{\prime}$ of the rhombus are identical with the patch of the hexagonal lattice shown in Fig. 1(a), where the centers of all hexagons are replaced by the states with allowed momenta. The number of these states is twice the number of hexagons. Note that the $y$ axis in Fig. 4(b) points in a direction opposite to that of the $y$ axis in Fig. 4(a). This is because the reciprocal lattice is the mirror image of the dual to the hexagonal lattice. The above construction offers a convenient way of building the first Brillouin zone of a tet- 
rahedral cage without the use of Eqs. (21), by taking the dual of its unfolded hexagonal patch.

\section{BAND STRUCTURE OF THE CAGE}

First consider a periodic lattice on the parent graphite sheet. The energy spectrum of this lattice in the tight-binding approximation is uniquely defined by the substitution of the allowed momenta [Eq. (21)] in the dispersion relation of graphene [Eq. (12)]. The trigonal lattice of states in the reciprocal space has a sixfold rotational symmetry around the origin. On the other hand, dispersion relation (12) has full $D_{6}$ symmetry. Indeed, $E_{\mu\left(k_{x}, k_{y}\right)}=E_{\mu\left(-k_{x}, k_{y}\right)}$ $=E_{\mu\left(k_{x},-k_{y}\right)}=E_{\mu\left(-k_{x},-k_{y}\right)}$ and direct calculation shows that $E_{\mu C_{6}\left(k_{x}, k_{y}\right)}=E_{\mu\left(k_{y}, k_{x}\right)}$, where $C_{6}$ is the rotation about $\pi / 3$. All the elements of the $D_{6}$ group are combinations of the above, energy-conserving transformations. Therefore, with some exceptions, the energies will occur in sextets or multiples thereof. The only exceptions to this rule are the six states $\boldsymbol{\Gamma}, \mathbf{M}_{1}, \mathbf{M}_{2}, \mathbf{M}_{3}, \mathbf{K}_{1}$, and $\mathbf{K}_{2}$ marked as black points in Fig. 2. Note that, except for $\mathbf{M}$ and $\mathbf{K}$ points, all states belonging to the three upper edges of the Brillouin zone also form sextets. This is because the center of each side is an allowed $\mathbf{M}$ state and any other state occurring on that side must have its mirror partner on the opposite side of $\mathbf{M}$ (by the symmetry of trigonal lattice) and two other, equal energy pairs on the remaining two edges. The center $\boldsymbol{\Gamma}$ is unique, and so the energy $\pm 3 \gamma$ is a singlet.

Now we introduce the extra tetrahedral boundary condition [Eq. (17)] which requires that antipodal points in $\mathbf{k}$ space be paired to yield even states. All sextets thus will be halved to triplets. In the tetrahedron the points $\mathbf{M}_{1}, \mathbf{M}_{2}$, and $\mathbf{M}_{3}$, considered in Sec. III, form a gerade triplet corresponding to the energy $-\gamma$. However, the degeneracy of this eigenvalue can be higher: all states on straight lines between neighboring $\mathbf{M}$ points and their antipodes (the dashed line in Fig. 2) belong to this eigenvalue. If we take, according to Eq. (21), the $\left(l_{1}, l_{2}\right)$ parametrization of allowed momenta $\mathbf{k}$ $=\mathbf{k}\left(l_{1}, l_{2}\right)$, then the $\mathbf{M}$ states are $\mathbf{M}_{1}=(-m, n), \quad \mathbf{M}_{2}$ $=(n, m+n)$, and $\mathbf{M}_{3}=(m+n, m)$. By simple geometrical considerations one can find the condition for the other states in the first Brillouin zone to belong to the dashed hexagon from Fig. 2. The necessary and sufficient condition for the $\left(l_{1}, l_{2}\right)$ pair to generate a $\mathbf{k}$ state, other than an $\mathbf{M}$ point, with eigenvalue $\pm \gamma$, is that

$$
m^{2}+n^{2}+m n=(m+n) l_{1}-n l_{2},
$$

where $l_{1}, l_{2}$, and $l_{1}-l_{2}$ belong to the interval $-(m+n)$ $<l_{1}, l_{2}, l_{1}-l_{2}<m+n$. One can observe that, e.g., the spectra for $m=n$ and $n=0$ have exact degeneracies $3(m-1)$ at $E=\gamma$ and $3+3(m-1)$ degeneracies at $E=-\gamma$.

The degeneracy at $E=0$, which corresponds to $\mathbf{K}$ points, is of special importance. These are the two points where both bands of the graphene sheet coincide and, at the half filling, meet with the Fermi energy. The symmetry of the reciprocal lattice implies that the degeneracy of the energy $E=0$ is either 2 or 0 . The necessary and sufficient condition for the occurrence of double degeneracy at $E=0$ can be again de- rived from Eq. (21), and it coincides with the condition that $m-n$ must be a multiple of three. The relation $m-n$ $\equiv 0 \bmod 3$ has a simple geometric interpretation, as it is exactly the condition that the $(\mathbf{3 , 6})$ cage $(m, n)$ should be a leapfrog. ${ }^{9}$ We will return to this aspect in a separate section on inflation relations.

Summarizing, we have shown that the spectrum of the tetrahedrally symmetric $(\mathbf{3 , 6})$ cage always includes $3 \gamma,-\gamma$, $-\gamma,-\gamma$, and always has a pseudobipartite character: two properties noted in Ref. 3 and proved there for a subset of the $(3, \mathbf{6})$ class. The spectrum has the form

$$
1+3 n_{1}+(2)+\left(3+3 n_{2}\right)+3 n_{3}
$$

where $n_{1}, n_{2}$, and $n_{3}$ are integers. In Eq. (25), 1 corresponds to an $E=3 \gamma$ singlet, $3 n_{1}$ are $n_{1}$ triplets (some of them may be multiples of 3 ) of energy $3 \gamma<E<0,3+3 n_{2}$ is the degeneracy of $E=-\gamma, 3 n_{3}$ are again $n_{3}$ triplets (with possible further degeneracy) of energy $0<E<-3 \gamma$, and 2 is the degeneracy of $E=0$ provided 3 divides $m-n$; otherwise this nonbonding level is absent. The pseudobipartite character matches the $n_{1}$ and $n_{2}+n_{3}$ triplets. Examples of the Brillouin zones and the spectra of cages corresponding to $m$ $=4$ and $n=0, \ldots, 4$ are shown in Fig. 5 .

The physical consequence of the above general pattern in the eigenvalue spectrum is that a $(\mathbf{3 , 6})$ cage of tetrahedral symmetry has a $\pi$ electronic configuration of one of two types, entirely determined by divisibility of $(m-n)$. Either the cage is a leapfrog, with $(m-n)=0 \bmod 3$, in which case $N$, the number of vertices, is a multiple of 12 , and the spectrum has $N / 2-2$ strictly bonding, two nonbonding and $N / 2$ strictly antibonding orbitals, and hence a closed shell configuration for the neutral carbon cage; or the cage is not a leapfrog, has $(m-n)=1 \bmod 3$, in which case $N$ is $4 \bmod 12$ and the spectrum has $N / 2-1$ strictly bonding and $N / 2+1$ strictly antibonding orbitals and hence a closed shell as the dication $C_{n}^{2+}$. The "extra" four atoms of the nonleapfrog case are to be found at the face centers of the master tetrahedron. A typical member of the $(\mathbf{3 , 6})$ class, if realized as a (strained) carbon cage, is therefore predicted to lose electrons easily, in contrast with the $(\mathbf{5 , 6})$ fullerenes which are electron-deficient in their chemistry. The electron deficiencies of the typical fullerene are rationalised by the presence in leapfrog $(\mathbf{5 , 6})$ cages of low-lying empty orbitals spanning the translational and rotational symmetries, and derived from the $\pi$ bonding orbitals of the 12 pentagons. ${ }^{10}$ The degenerate pair of orbitals at the Fermi energy, which is responsible for the opposite electron donating properties of $(\mathbf{3 , 6})$ cages, are related in an analogous way to the bonding orbitals of the four triangles.

\section{IRREDUCIBLE SYMMETRY REPRESENTATIONS OF THE TETRAHEDRAL EIGENVECTORS}

Having obtained the wave functions, it is always possible to determine their transformation under the symmetry elements of the point group of the cage. General rules can be given which relate the irreducible representations of the levels directly to their $\mathbf{k}$ values. In this section we will limit ourselves to the rule which decides the symmetry labels $A, E$, 

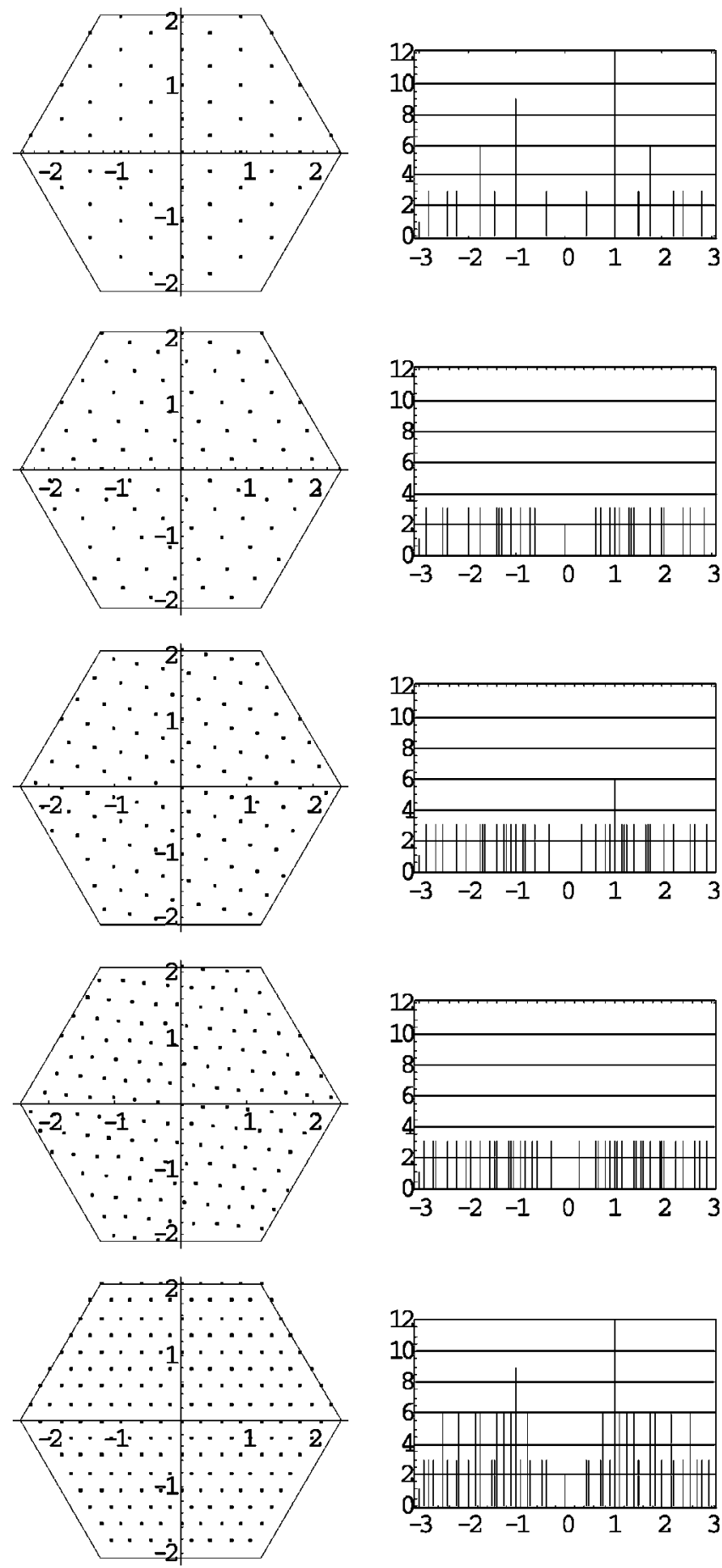

FIG. 5. Band structures and Brillouin lattice for cages with $m$ $=4$ and $n=0,1,2,3$, and 4 (from top to bottom). Energies are in units of $|\gamma|$. Note the twofold-degenerate nonbonding levels in the leapfrog cages $(4,1)$ and $(4,4)$. All spectra include the $(-3,+1$, $+1,+1)$ eigenvalues of the primitive tetrahedron.

and $T$ of the tetrahedral rotation group. The threefold axes of this group run through the vertices and face centers of the master tetrahedron. In Fig. 6(a) they are indicated on an unfolded cage. The origin of the hexagonal sheet itself coincides with a point of trigonal symmetry, which implies that there will also be a threefold axis through the center of the
Wigner-Seitz cell in reciprocal space. The $\boldsymbol{\Gamma}$ point itself thus corresponds to a totally symmetric state of $A$ symmetry. The doublet at $\mathbf{K}_{1}$ and $\mathbf{K}_{2}$ is always of $E$ symmetry. This can be shown as follows: an anticlockwise rotation of $\mathbf{K}_{1}$ in the diagram of Fig. 2 over $2 \pi / 3$ turns $\mathbf{K}_{1}$ into $\mathbf{K}_{1}-\mathbf{P}_{1}$. The action on the $\left|\mathbf{K}_{1}\right\rangle_{1, g}$ eigenvector thus reads

$$
\begin{aligned}
\hat{C}_{3}\left|\mathbf{K}_{1}\right\rangle_{1, g} & =\frac{1}{\sqrt{N}} \sum_{p} \exp \left[i s_{p} \mathbf{r}_{p} \cdot\left(\mathbf{K}_{1}-\mathbf{P}_{1}\right)\right] a_{p}^{+}|0\rangle \\
& =\exp \left(\frac{2 \pi i}{3}\right)\left|\mathbf{K}_{1}\right\rangle_{1, g} .
\end{aligned}
$$

To obtain this result it must be taken into account that the position vectors are expressed with respect to the coordinate origin in the center of an hexagon, i.e.,

$$
\begin{gathered}
\mathbf{r}_{p}=\frac{1}{3}\left(-\mathbf{T}_{1}+2 \mathbf{T}_{2}\right)+r \mathbf{T}_{1}+s \mathbf{T}_{2}, \quad \text { for black points, } \\
\mathbf{r}_{p}=\frac{1}{3}\left(\mathbf{T}_{1}+\mathbf{T}_{2}\right)+r \mathbf{T}_{1}+s \mathbf{T}_{2}, \quad \text { for white points, }
\end{gathered}
$$

where $r$ and $s$ are integers. The phase factor $\exp (2 \pi i / 3)$ is typical for a component of the twofold degenerate $E$ representation; the other component $\left|\mathbf{K}_{1}\right\rangle_{2, g}$ transforms as a complex conjugate.

The triplets in the spectrum are symmetrically displaced around the central $\boldsymbol{\Gamma}$ point. Under a $C_{3}$ rotation the three components will undergo a cyclic permutation, which implies that the character of a triplet vanishes; $\chi\left(C_{3}\right)=0$. As a result all triplets must have either $A+E$ or $T$ symmetry.

From the tetrahedral character table, these two possibilities can be distinguished by their character under any twofold rotation axis $C_{2}$ in $T$ :

$$
\begin{aligned}
& \chi^{A+E}\left(C_{2}\right)=3, \\
& \chi^{T}\left(C_{2}\right)=-1 .
\end{aligned}
$$

The $C_{2}$ axes run through the edge midpoints of the tetrahedron. On the unfolded lattice they form an interstitial triangulated lattice generated by the vectors $\mathbf{A} / 4$ and $\mathbf{B} / 4$ [gray spots in Fig. 6(b)].

If we require a given wave function to be symmetrical with respect to any $C_{2}$ axis, it must obey the following constraints:

$$
\begin{aligned}
& \Psi(\mathbf{r}+\mathbf{A} / 4)=\Psi(-\mathbf{r}+\mathbf{A} / 4)=\Psi(\mathbf{r}-\mathbf{A} / 4) \\
& \Psi(\mathbf{r}+\mathbf{B} / 4)=\Psi(-\mathbf{r}+\mathbf{B} / 4)=\Psi(\mathbf{r}-\mathbf{B} / 4)
\end{aligned}
$$

In both cases the first equality expresses total symmetry with respect to the $C_{2}$ axes, while the second equality expresses the even parity of the tetrahedral states with respect to the center of symmetry at the origin. By adding $\mathbf{A} / 4$ or $\mathbf{B} / 4$ in the arguments on the right and left of these equations, one obtains

$$
\begin{aligned}
& \Psi(\mathbf{r})=\Psi(\mathbf{r}+\mathbf{A} / 2), \\
& \Psi(\mathbf{r})=\Psi(\mathbf{r}+\mathbf{B} / 2),
\end{aligned}
$$

and, by induction, 


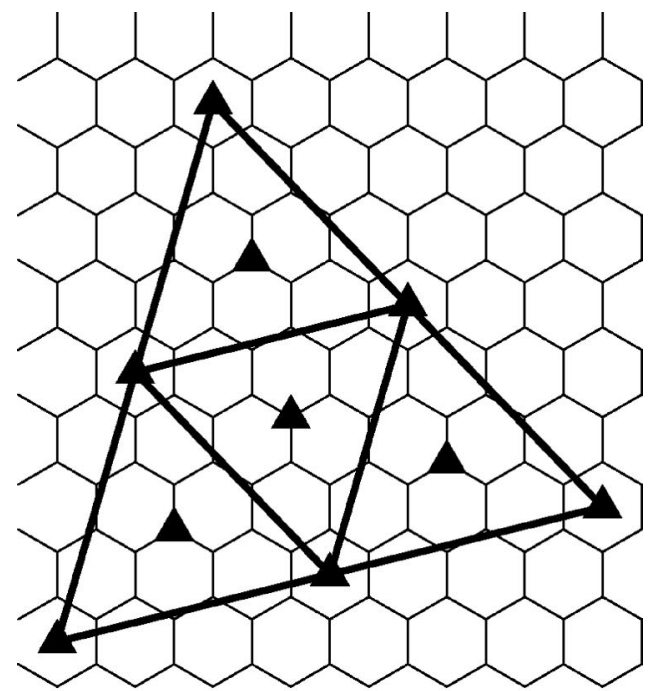

(a)

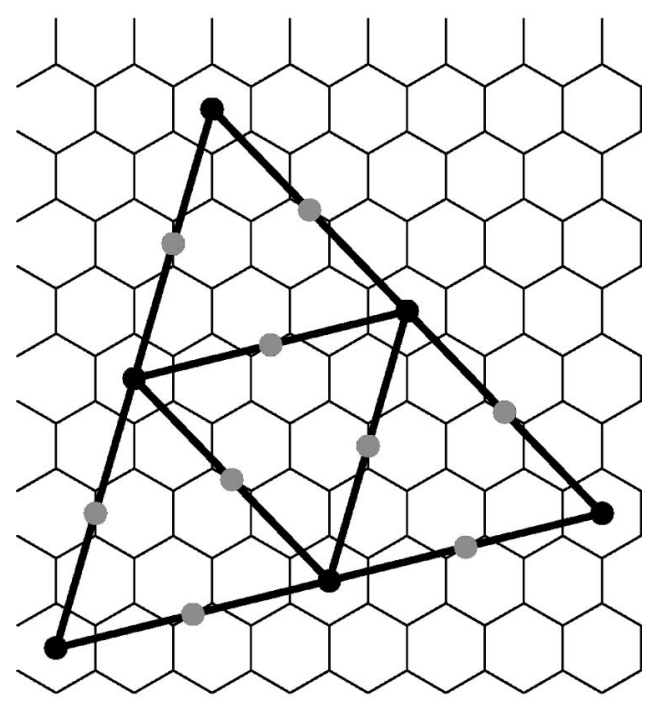

(b)

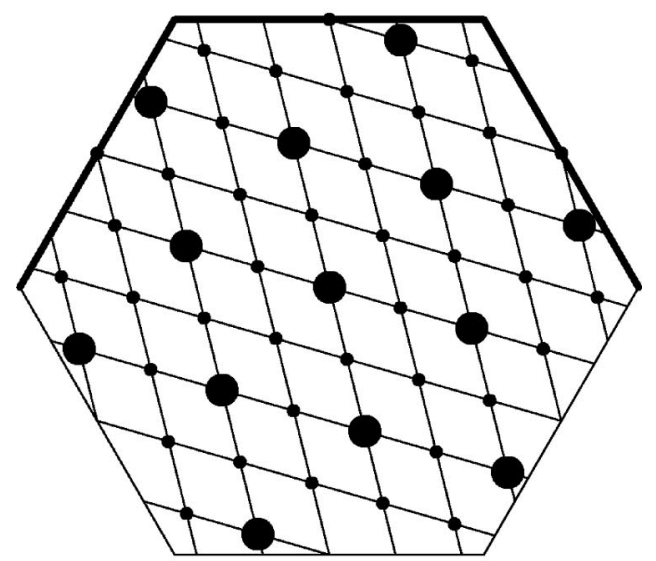

(c)

FIG. 6. Positions of the poles of the threefold (a) and twofold (b) symmetry axes of the tetrahedral cage of Fig. 1 in the unfolded patch. Heavy dots in (c) show the allowed $\mathbf{k}$ points with even indices $l_{1}$ and $l_{2}$ in the first Brillouin zone, which correspond to $A$ $+E$ triplets. The $\boldsymbol{\Gamma}$ point in the origin always belongs to the even lattice, and is totally symmetric.

$$
\Psi(\mathbf{r})=\Psi(\mathbf{r}+i \mathbf{A} / 2+j \mathbf{B} / 2) .
$$

Hence totally symmetric eigenvectors with respect to all $C_{2}$ axes must have periods that are only half the fundamental periods $\mathbf{A}$ and $\mathbf{B}$, i.e., they must fit into a torus that is four times smaller than the original one. The allowed grid points are determined by

$$
\begin{aligned}
& \mathbf{k} \cdot \mathbf{A} / 2=2 \pi l_{1}^{\prime}, \\
& \mathbf{k} \cdot \mathbf{B} / 2=2 \pi l_{2}^{\prime} .
\end{aligned}
$$

If we compare this result with the original zone-folding formula in Eq. (20) one obtains $l_{1}=2 l_{1}^{\prime}$ and $l_{2}=2 l_{2}^{\prime}$. Hence the $A+E$ triplets occur only at grid points with both $l_{1}$ and $l_{2}$ even. One-quarter of the total Brillouin grid is selected in this way.

As an example consider the tetrahedron with $m=3$ and $n=1$. This cage contains 52 atoms, spanning the following representation of $T$ :

$$
\Gamma=5 A+4 E+13 T .
$$

In Fig. 6(c) we show the allowed $\left(l_{1}, l_{2}\right)$ grid points in the Brillouin zone and also mark the $\left(2 l_{1}^{\prime}, 2 l_{2}^{\prime}\right)$ points by heavy dots.

The zone center $\boldsymbol{\Gamma}$ is of course part of the heavy dots, and corresponds to an $A$ state. The three $\mathbf{M}$ points are also present, but not at even grid positions; therefore they transform in this specific case as $T$. This leaves $4(A+E)$ and 12 $T$ triplets. We have indeed exactly $4 \times 3$ heavy dots and $12 \times 3$ light dots inside the zone, which identify $(A+E)$ and $T$ symmetries, respectively. Note that in general the $\mathbf{M}$ points need not be $T$ symmetric. As an example, for a cage which is twice as large as the one which we have just considered, the $\mathbf{M}$ points become even grid points and thus transform as $A+E$.

We briefly mention that one can also use similar techniques to determine the symmetry if the cage has $T_{d}$ symmetry. Each $\sigma_{d}$ symmetry plane evolves into two perpendicular symmetry planes when projecting the cage on the graphite sheet. In this way, one can distinguish between $A_{1}$ and $A_{2}$ representations, on the one hand, and between $T_{1}$ and $T_{2}$ representations on the other hand.

Simple counting of orbits (sets of equivalent positions) allows a complete breakdown of the eigenvector symmetries in all $T$ and $T_{d}(\mathbf{3 , 6})$ cages. Vertices of a $T[(m, n), m \neq n, n$ $\neq 0]$ cage fall into sets $O_{4}$ and $O_{12}$, and vertices of a $T_{d}$ cage $[(m, 0)$ or $(m, m)]$ fall into sets $O_{4}, O_{12}$, and $O_{24}$, all with their characteristic permutation symmetries. There are five cases:

(1) $T$ symmetry, leapfrog cage $\left(N=m^{2}+m n+n^{2}\right)$,

$$
\Gamma_{\sigma}(v)=(N / 3) A+(N / 3) E+N T
$$

(2) $T$ symmetry, nonleapfrog cage $\left(N=m^{2}+m n+n^{2}\right)$,

$$
\Gamma_{\sigma}(v)=\frac{N+2}{3} A+\frac{N-1}{3} E+N T .
$$

(3) $T_{d}$ symmetry, leapfrog cages, with $(m, 0)$, 


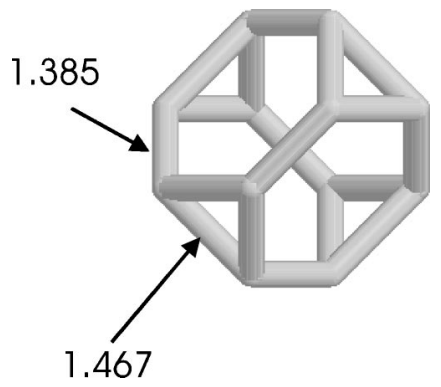

FIG. 7. Structure for the truncated tetrahedron $\mathrm{C}_{12}$ as obtained by DFT geometry optimization under $T_{d}$ constraints. A frequency analysis for this structure yields imaginary frequencies for one $T_{1}$ mode.

$$
\begin{aligned}
\Gamma_{\sigma}(v)= & \frac{m(m+3)}{6} A_{1}+\frac{m(m-3)}{6} A_{2}+\frac{m^{2}}{3} E+\frac{m(m-1)}{2} T_{1} \\
& +\frac{m(m+1)}{2} T_{2} .
\end{aligned}
$$

(4) $T_{d}$ symmetry, nonleapfrog cages with $(m, 0)$,

$$
\begin{aligned}
\Gamma_{\sigma}(v)= & \frac{(m+2)(m+1)}{6} A_{1}+\frac{(m-2)(m-1)}{6} A_{2} \\
& +\frac{(m+1)(m-1)}{3} E+\frac{m(m-1)}{2} T_{1} \\
& +\frac{m(m+1)}{2} T_{2} .
\end{aligned}
$$

(5) $T_{d}$ symmetry, leapfrog cages with $(m, m)$,

$$
\begin{aligned}
\Gamma_{\sigma}(v)= & \frac{m(m+1)}{2} A_{1}+\frac{m(m-1)}{2} A_{2}+m^{2} E \\
& +\frac{m(3 m-1)}{2} T_{1}+\frac{m(3 m+1)}{2} T_{2} .
\end{aligned}
$$

\section{COMPARISON WITH QUANTUM-CHEMICAL CALCULATIONS}

In order to test the validity of the simple tight-binding approximation, we have performed a standard densityfunctional theory (DFT) calculation on several tetrahedral (3, 6) cages. Initial coordinates were generated directly from the adjacency matrix, using the method of Fowler and Manolopoulos. ${ }^{11}$ The B3LYP functional was used, in combination with the split-valence basis sets from Schäfer et al. ${ }^{12}$ extended with a polarization function with exponent 0.80 . This DFT calculation was performed with the Turbomole code. $^{13}$

The smallest tetrahedral $(\mathbf{3 , 6})$ cage is the truncated tetrahedron $\mathrm{C}_{12}(m=1, n=1)$. This cage was optimized under $T_{d}$ symmetry constraints. Its geometry is shown in Fig. 7. The total binding energy per carbon atom is only $5.37 \mathrm{eV}$, indicating that this structure is less stable than a cyclic alternative. A frequency analysis reveals a negative Hessian eigen-

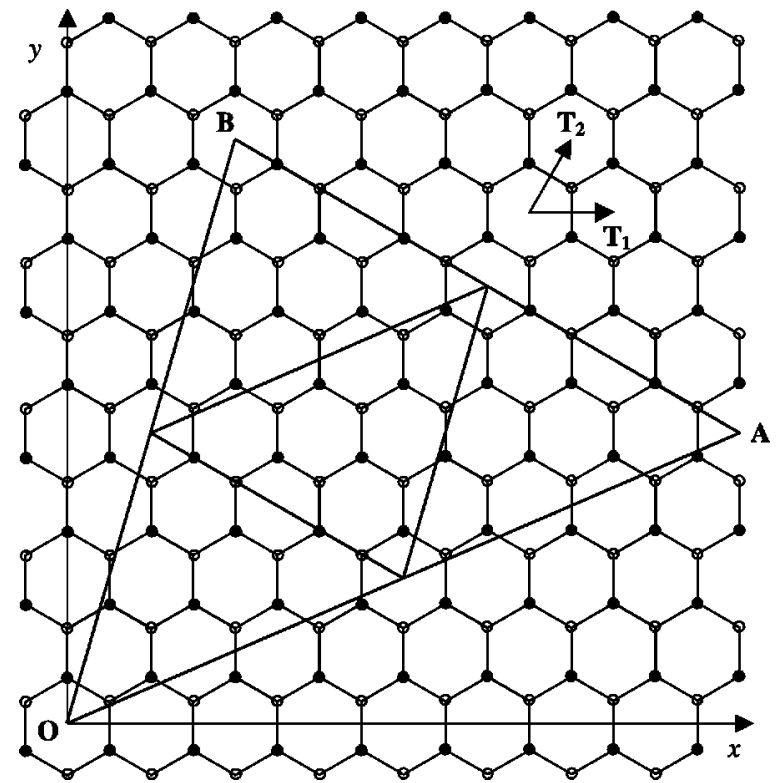

FIG. 8. Arbitrary $(\mathbf{3 , 6})$ cage, unfolded on the honeycomb plane. Indices correspond to $m=3, n=2, p=-1$, and $q=4$.

value for a normal mode with $T_{1}$ symmetry. Nevertheless the calculated frontiers orbitals are in agreement with the simple model predictions of the preceding sections. The highest occupied molecular orbital (HOMO) is a fully occupied $E$ level, as it should be for a leapfrog cage with $m-n$ $=0 \bmod 3$. The symmetries of the lowest unoccupied molecular orbital (LUMO) $\left(T_{2}\right)$ and next-LUMO $\left(T_{1}\right)$ are also as predicted. The calculated HOMO-LUMO gap is $1.93 \mathrm{eV}$, to be compared with $-\gamma$ in the model treatment.

Next we have investigated the $T_{d}$ cage $C_{48}$, which is again a leapfrog and has a closed shell as a neutral molecule. In this case the binding energy is equal to $6.59 \mathrm{eV}$ per carbon atom, while for graphite and fullerenes it is around 7.4 $\mathrm{eV}^{14,15}$ As expected, the larger value of the binding energy reflects that the strain in this larger cage is much relaxed as compared to $\mathrm{C}_{12}$. Arbitrary distortions of the cage, followed by optimization without symmetry restriction, always restored the tetrahedral structure, indicating that it is now a stable minimum. Furthermore, the symmetries of the frontier orbitals - from $\mathrm{HOMO}-2$ to $\mathrm{LUMO}+1$ - are as predicted by the tight-binding model.

Finally we have also investigated the $\mathrm{C}_{52}$ cage with $T$ symmetry shown in Fig. 1(b). Since this cage is not a leapfrog $(m=3, n=1)$ it has a closed shell as a dication, so the DFT calculation was made for $\mathrm{C}_{52}{ }^{2+}$. Optimization under a tetrahedral symmetry constraint yielded the folded geometry shown in Fig. 1(b). In the neutral state the cage was found to be subject to Jahn-Teller distortions, in line with a $t^{2}$ groundstate configuration. However the closed-shell dication is confirmed to remain stable with respect to symmetry-lowering distortions. Orbital energies and symmetries were calculated, and compared to the tight-binding results. The parameters $\alpha$ and $\gamma$ of the $\pi$-electron Hamiltonian were fitted to reproduce the calculated HOMO and LUMO levels $(\alpha=-11.64 \mathrm{eV}$ and $\gamma=2.13 \mathrm{eV}$ ). In Table I we list the results for eight triplets in the vicinity of the Fermi level. The symmetries of the 
TABLE I. Orbital energies and symmetries for $\mathrm{C}_{52}{ }^{2+}$ calculated by DFT and compared to a $\pi$-electron Hamiltonian; tight-binding HOMO and LUMO were fitted to DFT results.

\begin{tabular}{|c|c|c|c|c|}
\hline \multicolumn{2}{|c|}{$\begin{array}{c}\text { DFT } \\
\text { symmetry energy }(\mathrm{eV})\end{array}$} & & \multicolumn{2}{|c|}{$\begin{array}{l}\text { Tight-binding } \\
\text { symmetry energy }(\mathrm{eV})\end{array}$} \\
\hline$T$ & -14.7747 & & $T$ & -14.7869 \\
\hline$T$ & -14.0722 & & $T$ & -14.1801 \\
\hline$E$ & -13.1856 & & \multirow{2}{*}{$E+A$} & \multirow{2}{*}{-13.4218} \\
\hline A & -13.0710 & & & \\
\hline$T$ & -12.7683 & HOMO & $T$ & -12.7683 \\
\hline$T$ & -10.5202 & LUMO & $T$ & -10.5202 \\
\hline E & -9.8103 & & \multirow{2}{*}{$E+A$} & \multirow{2}{*}{-9.8667} \\
\hline A & -9.4753 & & & \\
\hline$T$ & -8.9673 & & $T$ & -9.51545 \\
\hline$T$ & -8.6185 & & $T$ & -9.10837 \\
\hline
\end{tabular}

frontier orbitals are as predicted by the tight-binding model. In addition a very satisfactory agreement of energies is noted. Similar correspondences have frequently been noted for fullerenes and nanotubes, and explain the success of simple two-parameter tight-binding models for carbon clusters.

\section{GENERALIZATION TO ARBITRARY $(3,6)$ CAGES}

The construction can easily be generalized to the arbitrary $(3,6)$ cage. ${ }^{1}$ The net is defined by the two vectors

$$
\begin{aligned}
& \mathbf{A}=2 m \mathbf{T}_{1}+2 n \mathbf{T}_{2}, \\
& \mathbf{B}=2 p \mathbf{T}_{1}+2 q \mathbf{T}_{2},
\end{aligned}
$$

where $m, n, p$, and $q$ are integer parameters, and $m q-p n$ $\neq 0$. The number of nodes of such a cage is $N=4(m q$ $-p n)$. The example of a cage corresponding to $(m, n, p, q)$ $=(3,2,-1,4)$ is shown in Fig. 8. The construction of the wave function by the use of the covering function resembles that described in Sec. III. The only difference is that in the tiling of the plane, shown in Fig. 3, the equilateral triangles are now replaced by arbitrary triangles defined by $\mathbf{A}$ and $\mathbf{B}$.

To find the electronic structure of an arbitrary $(\mathbf{3 , 6})$ cage we again use conditions (20), assuring the periodicity of the covering function. The solution for the allowed $\mathbf{k}$ vectors is now

$$
\begin{gathered}
k_{x}=\frac{\pi}{\sqrt{3} a} \frac{q l_{1}-n l_{2}}{m q-p n}, \\
k_{y}=\frac{\pi}{3 a}\left[\frac{-(2 p+q) l_{1}+(2 m+n) l_{2}}{m q-p n}\right] .
\end{gathered}
$$

Such a general cage has at least $D_{2}$ symmetry. The corresponding irreducible representations can easily be determined. The $\boldsymbol{\Gamma}$ and $\mathbf{K}$ points will always be of $A$ type.

The neutral cage will be a closed shell with two filled nonbonding orbitals if simultanously $m-n=0 \bmod 3$ and $p-q=0 \bmod 3$ (i.e., a leapfrog). Otherwise there will be a closed shell as dication. Note that for a tetrahedral cage both conditions coincide, since $p=-n$ and $q=m+n$.

\section{INFLATION RELATIONS}

Consider a general transformation of the $(m, n)$ indices of a tetrahedral cage,

$$
\left(m^{\prime}, n^{\prime}\right)=(m, n)\left(\begin{array}{ll}
\alpha & \beta \\
\gamma & \delta
\end{array}\right),
$$

where $\alpha, \beta, \gamma$, and $\delta$ are integers. We call such a transformation an inflation if the number of atoms in the new cage with indices $\left(m^{\prime}, n^{\prime}\right)$ is a multiple of the number of atoms in the original cage. For this to be true for all $m$ and $n$, it is required that

$$
\alpha^{2}+\alpha \beta+\beta^{2}=\gamma^{2}+\gamma \delta+\delta^{2}=2 \alpha \gamma+\alpha \delta+\gamma \beta+2 \beta \delta
$$

These relations can be turned into a quadratic equation for $\gamma$ as a function of $\alpha$ and $\beta$,

$$
\gamma^{2}-\alpha \gamma-(\alpha+\beta) \beta=0,
$$

with two independent solutions $\gamma=-\beta$ and $\gamma=\alpha+\beta$. The fourth parameter $\delta$ then corresponds to $\delta=\alpha+\beta$ and $\delta$ $=-\alpha$, respectively. The first solution describes the inflation of the cage as a matrix transformation of type

$$
\left(m^{\prime}, n^{\prime}\right)=(m, n)\left(\begin{array}{cc}
\alpha & \beta \\
-\beta & \alpha+\beta
\end{array}\right) .
$$

The vectors $\mathbf{A}^{\prime}=2 m^{\prime} \mathbf{T}_{1}+2 n^{\prime} \mathbf{T}_{2}$ and $\mathbf{B}^{\prime}=2\left(m^{\prime}+n^{\prime}\right) \mathbf{T}_{2}$ $-2 n^{\prime} \mathbf{T}_{1}$, which define the cage, can easily be expressed as linear combinations of $\mathbf{A}$ and $\mathbf{B}$ vectors of the parent cage:

$$
\left(\mathbf{A}^{\prime}, \mathbf{B}^{\prime}\right)=(\mathbf{A}, \mathbf{B})\left(\begin{array}{ll}
\alpha & -\beta \\
\beta & \alpha+\beta
\end{array}\right) .
$$

An immediate consequence of this relationship is that the spectrum of eigenvalues of the cage $\mathbf{O A B}$ is entirely contained in the spectrum of eigenvalues of the inflated cage $\mathbf{O A}^{\prime} \mathbf{B}^{\prime}$ for any inflation obeying Eq. (45). Indeed consider an eigenvector $\mathbf{k}$ which is periodic over $\mathbf{O A}$ and $\mathbf{O B}$; then by combining Eqs. (20) and (46), one immediately verifies that it will also be periodic over $\mathbf{O A}^{\prime}$ and $\mathbf{O B}^{\prime}$ :

$$
\begin{gathered}
\mathbf{k} \cdot \mathbf{A}^{\prime}=2 \pi\left(l_{1} \alpha+l_{2} \beta\right), \\
\mathbf{k} \cdot \mathbf{B}^{\prime}=2 \pi\left[-l_{1} \beta+l_{2}(\alpha+\beta)\right] .
\end{gathered}
$$

This implies that $\mathbf{k}$ is also an allowed eigenvector of the inflated cage, which proves the subspectrality of the parent cage. An important example of this type of inflation is the so-called leapfrog transformation, corresponding to $\alpha=1$ and $\beta=1$.

$$
\left(m^{\prime}, n^{\prime}\right)_{\mathcal{L}}=(m, n)\left(\begin{array}{cc}
1 & 1 \\
-1 & 2
\end{array}\right)
$$


The leapfrog $\mathcal{L}$ of a spherical trivalent polyhedron on $v$ vertices $P_{v}$ is constructed by first omnicapping $P_{v}$ and then taking the dual of the result. $\mathcal{L}\left(P_{v}\right)$ has $3 v$ vertices and if $P_{v}$ is a $(\mathbf{q}, \mathbf{6})$ cage, then so is $\mathcal{L}$. General results about the spectrum of $\mathcal{L}\left(P_{v}\right)$ can be proved. If $P_{v}$ has at least one face of size not divisible by 3 , the spectrum of $\mathcal{L}\left(P_{v}\right)$ has $3 v / 2$ strictly positive and $3 v / 2$ strictly negative eigenvalues. ${ }^{16}$ If all faces of $P_{v}$ are of sizes divisible by 3 , the spectrum has 2 zero and $3 v / 2-2$ strictly positive eigenvalues. ${ }^{16,17}$ The latter result includes $(\mathbf{3 , 6})$ leapfrog cages as a special case.

A further example is the quadrupling or chamfering ${ }^{18}$ transformation which is generated by $\alpha=2$ and $\beta=0$ :

$$
\left(m^{\prime}, n^{\prime}\right)_{\mathcal{Q}}=(m, n)\left(\begin{array}{ll}
2 & 0 \\
0 & 2
\end{array}\right) .
$$

This expansion corresponds to the bevelling of every edge. In this way, every edge is replaced by an hexagon. For both examples the spectrum of the parent $(\mathbf{3 , 6})$ cage is contained in the spectrum of the tripled or quadrupled cage.

The second solution of Eq. (44) is of type

$$
\left(m^{\prime}, n^{\prime}\right)=(m, n)\left(\begin{array}{cc}
\alpha & \beta \\
\alpha+\beta & -\alpha
\end{array}\right) .
$$

In this case the relationship between the triangles $\mathbf{O A B}$ and $\mathbf{O A}^{\prime} \mathbf{B}^{\prime}$ is more involved. One has

$$
\left(\mathbf{A}^{\prime}, \mathbf{B}^{\prime}\right)=(\mathbf{A}, \mathbf{B}) \frac{1}{m^{2}+n m+n^{2}}\left(\begin{array}{cc}
\alpha m(m+2 n)+\beta n(2 m+n) & \alpha n(2 m+n)-\beta\left(m^{2}-n^{2}\right) \\
-\alpha n(2 m+n)+\beta\left(m^{2}-n^{2}\right) & \alpha\left(m^{2}-n^{2}\right)+\beta m(m+2 n)
\end{array}\right) .
$$

As the coefficients in this expression are fractional numbers, subspectrality no longer holds in this case.

Inflation can also be applied to the $(\mathbf{3 , 6})$ cages of $D_{2}$ symmetry. Here we will limit ourselves to cages for which subspectrality exists. This implies that the inflated cage $\mathbf{O A}^{\prime} \mathbf{B}^{\prime}$ is obtained by a linear transformation with integer coefficients such as:

$$
\left(\mathbf{A}^{\prime}, \mathbf{B}^{\prime}\right)=(\mathbf{A}, \mathbf{B})\left(\begin{array}{ll}
\kappa & \lambda \\
\mu & \nu
\end{array}\right) .
$$

The multiplication factor in this case is given by $\kappa \nu-\lambda \mu$. The corresponding transformation of the indices reads

$$
\left(m^{\prime}, n^{\prime}, p^{\prime}, q^{\prime}\right)=(m, n, p, q)\left(\begin{array}{cccc}
\kappa & 0 & \mu & 0 \\
0 & \kappa & 0 & \mu \\
\lambda & 0 & \nu & 0 \\
0 & \lambda & 0 & \nu
\end{array}\right) .
$$

Note that leapfrogging is not included in this transformation.

\section{CONCLUSIONS}

By the introduction of suitable topological defects the hexagonal lattice can be wrapped up to a polyhedral cage. In the case of four triangular defects one obtains a network molecule that can be laid over a tetrahedron or bisphenoid. As we have shown in this paper, the electronic structure of such $(3, \mathbf{6})$ cages is very close to the band structure of the parent honeycomb sheet. A straightforward projection method relates this sheet to nanotubes, the nanotubes to toroids, and finally the toroids to tetrahedral cages. The first two steps involve zone folding in two different directions, and the final step removes half of the toroidal spectrum by symmetry adaptation to a centrosymmetric gluing condition. As a result, closed-form expressions are available for the solutions of the $\pi$-electron Hamiltonian in any $(\mathbf{3 , 6})$ cage. These confirm and generalize previously observed subspectrality relationships. ${ }^{1}$.

The analysis indicates that the $(\mathbf{3 , 6})$ cages can be divided into two families, leapfrogs and nonleapfrogs, depending on whether the differences between the cage indices $m-n$ and $p-q$ are divisible by 3 or not. This distinction yields a simple criterion for the electronic ground state: leapfrogs have a closed-shell ground state in the neutral form, while nonleapfrogs are closed shells as di-cations. Furthermore leapfrogs always have two nonbonding highest occupied molecular orbitals, which can easily act as electron donor levels. So far no molecular realizations of such cages have been reported. It is expected that triangular defects will cause a lot of strain in a carbon network. Nonetheless the parent tetrahedral $C_{4}$ cage $(m=1, n=0)$ already exists in saturated form as a tetrahedrane, $C_{4} R_{4}$, where $R$ represents a bulky tertiobutyl substituent. ${ }^{19}$ DFT calculations show that the smallest truncated tetrahedron $C_{12}$ is unstable, whereas higher networks such as $C_{48}$ and $C_{52}$ are stable which is in agreement with the expectation that the strain is less in larger cages. This makes them realistic targets for chemical synthesis.

\section{ACKNOWLEDGMENTS}

The Leuven team ackowledges financial support from the Flemish National Science Foundation (FWO) and from the Belgian Government through the concerted action scheme. The Katowice team acknowledges support from the Polish State Committee for Scientific Research (KBN) under Grant No. 5P03B03320. 
*Corresponding author.

${ }^{1}$ P. W. Fowler and J. E. Cremona, J. Chem. Soc., Faraday Trans. 93, 2255-2262 (1997).

${ }^{2}$ M. Goldberg, Tohoku Math. J. 43, 104 (1937).

${ }^{3}$ P. W. Fowler, P. E. John, and H. Sachs, $(\mathbf{3}, \mathbf{6})$ cages, Hexagonal toroidal cages and their spectra, DIMACS Series in Discrete Math. Theoret. Comput. Sci. 51, 139-174 (2000).

${ }^{4}$ J. González, F. Guinea, and V. A. H. Vozmediano, Nucl. Phys. B 406, 771 (1993).

${ }^{5}$ P. W. Fowler and D. E. Manolopoulos, An Atlas of Fullerenes (Clarendon Press, Oxford, 1995).

${ }^{6}$ S. L. Altmann, Band Theory of Solids: an Introduction from the Point of View of Symmetry (Clarendon Press, Oxford, 1991).

${ }^{7}$ P. R. Wallace, Phys. Rev. 71, 622 (1947).

${ }^{8}$ A. Ceulemans, L. F. Chibotaru, S. Bovin, and P. W. Fowler, J. Chem. Phys. 112, 4271 (2000).

${ }^{9}$ P. W. Fowler and J. I. Steer, J. Chem. Soc. Chem. Commun. 1997, 1403.

${ }^{10}$ P. W. Fowler and A. Ceulemans, J. Phys. Chem. 99, 508 (1995).
${ }^{11}$ D. E. Manolopoulos, P. W. Fowler, J. Chem. Phys. 96, 7603 (1992).

${ }^{12}$ A. Schäfer, H. Horn, and R. Ahlrichs, J. Chem. Phys. 97, 2571 (1992).

${ }^{13}$ R. Ahlrichs, M. Bär, M. Häser, H. Horn, and C. Kölmel, Chem. Phys. Lett. 162, 165 (1989).

${ }^{14}$ W. Ebbesen, Carbon Nanotubes: Preparation and Properties (CRC Press, Boca Raton, FL, 1997).

${ }^{15}$ M. S. Dresselhaus, G. Dresselhaus, and P. C. Eklund, Science of Fullerenes and Carbon Nanotubes (Academic Press, New York, 1994).

${ }^{16}$ D. E. Manolopoulos, D. R. Woodall, and P. W. Fowler, J. Chem. Soc., Faraday Trans. 88, 2427 (1992).

${ }^{17}$ P. W. Fowler and K. M. Rogers, J. Chem. Soc., Faraday Trans. 94, 2509 (1998).

${ }^{18}$ M. Goldberg, Tohoku Math. J. 40, 226 (1935).

${ }^{19}$ G. Maier, S. Pfriem, U. Schaefer, K.-D. Malsch, and R. Matusch, Chem. Ber. 114, 3965 (1981). 